\title{
In vitro transforming potential, intracellular signaling properties and sensitivity to a kinase inhibitor (sorafenib) of RET proto-oncogene variants Glu511Lys, Ser649Leu and Arg886Trp
}

\author{
Hugo Prazeres1,2,3, Joana P Couto', Fernando Rodrigues4, João Vinagre', \\ Joana Torres', Vitor Trovisco', Teresa C Martins², Manuel Sobrinho-Simões ${ }^{1,3}$ and \\ Paula Soares 1,3 \\ IInstitute of Molecular Pathology and Immunology of the University of Porto (IPATIMUP) - Cancer \\ Biology, Porto, Portugal \\ 2Molecular Pathology Service, Portuguese Institute of Oncology of Coimbra FG, Coimbra, Portugal \\ 3Department of Pathology, Medical Faculty, University of Porto, Porto, Portugal \\ ${ }^{4}$ Endocrinology Service, Portuguese Institute of Oncology of Coimbra FG, Coimbra, Portugal
}

Correspondence should be addressed to $P$ Soares

Email

psoares@ipatimup.pt

The authors and journal apologise for an omission in the above paper, which appeared in volume 18 part 4 , pages 401-412. The text 'This work was also supported by FEDER funds through Programa Operacional Factores de Competitividade - COMPETE (FCOMP-01-0124-FEDER 011267).' was omitted from the Funding section given on page 409.

The correct Funding section is given in full below:

\section{Funding}

The authors would like to acknowledge funding from grants from the Portuguese Foundation for Science and Technology (SFRH/BD/30041/2006 and PTDC/SAU-OBD/101242/2008), the Portuguese Society of Endocrinology and Metabolism (Edward Limber Prize) and the Portuguese Ministry of Health (13/2007). IPATIMUP is an associated Laboratory of the Portuguese Ministry of Science, Technology and Higher Education and is partially supported by the Portuguese Foundation for Science and Technology. This work was also supported by FEDER funds through Programa Operacional Factores de Competitividade - COMPETE (FCOMP-01-0124-FEDER 011267). 